\title{
Haptic Structuring Assistive Innovation for Individuals Who Are Visually Impaired
}

\author{
Vivek Agrawal, Vishakha Singh
}

\begin{abstract}
Powerful correspondence for the visually impaired can be upgraded by haptic interfaces with the world around individuals living with visual deficiency. This writing audit will endeavor to respond to extreme inquiries concerning where haptic innovation is going throughout the following decade predicted by an overview of bleeding edge haptic innovation blended in with a discourse of the upsides and downsides of deliberately chose high effect research articles at last, hypothesizing the eventual fate of haptics through designing patterns and development of arrangement changes.
\end{abstract}

Keywords: Haptic, Technology, Touch, Visually Impaired

\section{INTRODUCTION}

$\mathrm{V}$ isual inabilities obstruct a person's capacity to carry on an important life in a general public where the transcendent method of correspondence is through the feeling of sight. Insights through the World Health Organization (WHO) gauge that somewhere in the range of 285 million individuals worldwide are vision-hindered, and of that, 39 million are visually impaired with 1.4 million of them being youngsters (Niu, 2013; WHO, 2014). Turning a center onto the United States, visual impedance had a commonness of 19 million (National Federation of the Blind, 2009) toward the beginning of the 21st century, of which 1.3 million are visually impaired. Incorporated into the 1.3 million are 53,600 youngsters and youths who are dazzle (International, 2014). Youngsters with visual weakness are in danger for encountering troubles in language advancement (Bigelow, 1987; McConachie, 1990; Preisler, 1995). One apparatus worked viably to battle lack of education in the visually impaired network are strategies that include haptic learning (Barlow-Brown and Connelly, 2002). \The most mainstream type of haptic learning is the character coding of Braille which employments raised specks inside a rectangular fix embellished on paper encoding the letters in order and accentuation. Barlow-Brown 2002 (Barlow-Brown and Connelly, 2002) did an examination connecting Braille to improved perusing of visually impaired kids over kids that don't learn Braille.

Revised Manuscript Received on September 15, 2020.

* Correspondence Author

Vivek Agrawal*, B.Tech, Student, Vellore Institute of Technology, Vellore, (Tamil Nadu), India. E-mail: agrawal.vivek2017@gmail.com

Vishakha Singh, B.Tech, Student, Vellore Institute of Technology, Vellore E-mail: vishakha.singh1905@gmail.com

(C) The Authors. Published by Blue Eyes Intelligence Engineering and Sciences Publication (BEIESP). This is an open access article under the CC BY-NC-ND license (http://creativecommons.org/licenses/by-nc-nd/4.0/)

\section{METHODS}

The research community points out the importance of usability in the product development lifecycle and stressed the need for usability testing to be performed throughout the product development process, starting from prototype testing based on various techniques for visually impaired people. When evaluating the user product interaction, user activities are coordinated with a complex combination of sensory stimuli that is recorded via different sensory channels, such as touch, vision and hearing. In order for results to be validated, it is necessary that the prototypes simulate the actual behavior and the appearance of the product being developed, and this ability is closely linked to the performance capability of simulation technologies, both hardware and software (Ferrise, Bordegoni \& Graziosi, 2013). The technological development in different environments, specifically virtual and augmented reality, for blind people, has led to the development of more intuitive interfaces for design (Shen, Ong \& Nee, 2010), enabling the implementation of users testing activities from the interaction with different prototypes.

\section{WHAT IS HAPTIC}

Haptics is comprehended to mean a detecting and control through touch. Since the early piece of twentieth century analysts utilized the word haptics for concentrates on the dynamic bit of substantial items. In the late nineteen-eighties, when research started in the beginning times of mechanical technology wherein sensors encouraged counterfeit touch from which grew a requirement for a name for another order and "haptics" was re-imagined (Srinivasan, 1995). One underestimates the significance of the feeling of touch for state tying a shoe ribbon or turning a water valve. These activities would turn out to be about unimaginable without the important tactile input. Consider attempting to bite with Novocain in your jaw muscle, totally ignorant of where your cheeks are when biting. In present day innovations, engineers endeavor to improve controller execution in remote reenacted applications and that is the place haptic plans becomes possibly the most important factor empowering an administrator to broaden the utilization of ones material capacity past an arm's compass (Hannaford and Okamura, 2008). The human haptic framework is characterized to incorporate the whole tangible, engine and intellectual segments of the body-cerebrum framework. Specifically, when we contact an item with our hand the whole haptic framework, the nerves joined to our muscles (kinaesthetic commitment)

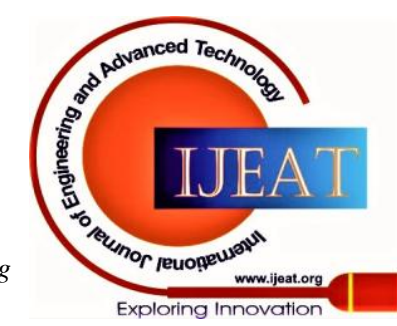




\section{Haptic Structuring Assistive Innovation for Individuals Who Are Visually Impaired}

and the nerves lying just underneath the skin layering the hand (cutaneous commitment), and cognizance work as one the give the engine control framework relevant criticism so as to procure fine engine aptitudes. Material gadgets interface with the haptic framework as the extending and pulling impacts on the skin surface which in term bother our inherent haptic sensors. (Demain, Metcalf, Merrett, Zheng, and Cunningham, 2013). Table 1 underneath records basic terms and there definitions utilized in discussion related tohaptic structure. This data can a helpful reference for perusers new to the innovation.

\section{TABLE 1: Terminology of haptics}

\begin{tabular}{|l|l|}
\hline Haptic & $\begin{array}{l}\text { Relating to the sense of touch in all } \\
\text { of its forms, including those below }\end{array}$ \\
\hline Proprioceptive & $\begin{array}{l}\text { Relating to sensory information } \\
\text { about the state of the body } \\
\text { (includingcutaneous, kinaesthetic, } \\
\text { and vestibular sensations) }\end{array}$ \\
\hline Vestibular & $\begin{array}{l}\text { Pertaining to the perception of } \\
\text { head position, acceleration, and } \\
\text { deceleration }\end{array}$ \\
\hline Kinaesthetic & $\begin{array}{l}\text { Meaning the feeling of motion. } \\
\text { Relating to sensations originating } \\
\text { in muscles, tendons, and joints }\end{array}$ \\
\hline Cutaneous & $\begin{array}{l}\text { Pertaining to the skin itself or the } \\
\text { skin as a sense organ. Includes } \\
\text { sensation ofpressure, temperature, } \\
\text { and pain }\end{array}$ \\
\hline Tactile & $\begin{array}{l}\text { Pertaining to the cutaneous sense } \\
\text { but more specifically to the } \\
\text { sensation of pressure rather than } \\
\text { temperature or pain }\end{array}$ \\
\hline $\begin{array}{l}\text { Force } \\
\text { feedback }\end{array}$ & $\begin{array}{l}\text { Relating to the mechanical } \\
\text { production of information }\end{array}$ \\
\hline
\end{tabular}

\section{MATERIAL BASED CORRESPONDENCE PARADIGM REQUIRED}

As the writing states, a dire need exists for either grasping another type of material learning past Braille (Niu, 2013; Shelton, 2012) or put into research that will prompt inexpensively mass created Braille perusers, e.g., MEMS actuator innovation, that will propel the material interface into the 21st century as exhibited in a paper by Xie 2014 at MIT (Xie, Zaitsev, Vel?squez-Garc?a, Teller, and Livermore, 2014). Carnegie Mellon College (CMU) has been taking a shot at a haptic showcase since 2010 that utilizations electrovibration in a gadget they call TelsaTouch. (Bau, Poupyrev, Israr, and Harrison, 2010). This gadget can copy an assortment of surfaces through utilizing a paper-slim film layered on a touchscreen glass board, to make the electrovibration correspondence medium doubly intelligent in an extremely powerful manner, as will be clarified later in the paper.(Bau et al., 2010). Perhaps the best resource as a scholarly foundation is creating innovation that is PC based and has handy applications based intensely on inspecting human factors that upgrade administrator ease of use. For example CMU's Personal satisfaction and Innovation (QoLT) (Carnegie_Melon_Univeristy, 2014) is exceptionally touted for making significant commitments through their revelation of imaginative arrangements that improve the lives of individuals with incapacities (PWD).

\section{SENSOR TECHNOLOGY IN HAPTIC DEVICES}

Structuring exact sensors to copy the capacity of the mechanoreceptors is an overwhelming building accomplishment. On the off chance that designers can achieve this, we can effectively figure out the components in our body that take into consideration the transmission of data through the feeling of contact. Braille for instance uses human's amazing capacity to transmit data through our fine feeling of touch at the tips of our fingers. As referenced before the fingertips have the most thickly populated plan of receptors in our whole body, meaning how much this tactile apparatus brings to the table AT engineers for the visually impaired. (Demain et al., 2013). In spite of the fact that we have the capacity to feel the state of letter set lettering decorated on paper (i.e., not encoded in Braille be that as it may, genuinely embellished letters), some specific letters contrast by such a little degree (e.g., "F" and "E") that raised letters would need to be huge so as to permit the "sharpness" of our finger tip's to observe between them. (Summers and Chanter, 2002)

\section{GRAPHICAL USER INTERFACE FOR THE BLIND}

Assistive technologists battle to discover correspondence modes for individuals who are oblivious in regards to make a gadget to repeat a visual data into a material medium.Translating the visual data encoded in the 2-D universe of drawings and symbols are absent in interpretation past content encoding with Braille. A perfect material interface would join the capacity to give both literary and graphical data in a single singular motion. One pattern in AT gadgets is pushing toward dynamic graphical material showcases. Think about the hindrances for the oblivious in regards to get to the web. Specialists are chipping away at finding a material graphical UI (Schiewe, Köhlmann, Nadig, and Weber, 2009) and making that one stride further presenting material symbols (Pietrzak, Crossan, Brewster, Martin, and Pecci, 2009) that will decipher non-content exchange. At last the objective is build up a medium that will be an all inclusive plan that would help the impaired populace as well as, e.g., incorporate into an augmented simulation gaming stage (Thorner and Heilman III, 2002). One innovative pattern in material showcases are as a two dimensional presentation (e.g., contact screen) that is jolted to give the hand contact receptors the dream of a finished surface, a surface with a third measurement. This gadget is called an electro-material showcase.

\section{FUTURE HAPTIC DESIGNS}

Despite the fact that, the specialists making this translational science have made astonishing advances in the course of the most recent couple of decades with innovations like refreshable Braille perusers (assistive innovation answer to the iPad for located individuals), the obstruction to future advances is engineers powerlessness to create a little enough actuator, the core of the refreshable Braille peruser (could be called Refreshable Braille Display). These little actuators are what raise and lower the material surface, similar to the "pen" in our "pen a paper," as it were,

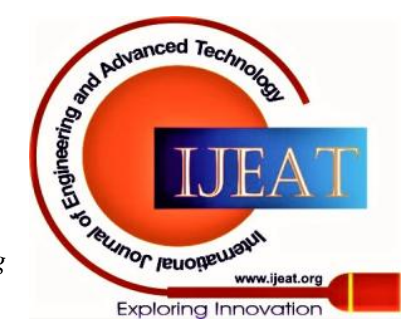


with regards to imparting through material surfaces. With regards to making a beneficial haptic interface it is the fine separating in the stick (actuator driven) clusters that will bringing about the requirement for little actuators, the thistle in the side of haptic plan engineers.(Kurt A Kaczmarek, Webster, Bach-y-Rita, and Tompkins, 1991; Leung, MacLean, Bertelsen, and Saubhasik, 2007; Lévesque).

In the event that we investigate the refreshable Braille show just, it will be modest and solid actuators that will consider large scale manufacturing of these things conceivable at a sensible expense to the purchaser. In any case, without a ton of R\&D cash, inventive advancement is moderate. Scientists have been attempting to escape from actuators with too many moving parts. One response to this issue is actuators made of formed memory composite (SMA) that when accused of an electrical flow a structure state change happens because of an expansion in temperature. On the off chance that their unheated shape is a curl, the loop may loosen up to stretch out their shape to potentially make a dimple in the haptic surface. The one downside is the time it takes to warm, a defer that is baffling to the client (Mizukami and Sawada, 2006). Additionally, the space issue concerning firmly stuffed fairly packed actuators underneath the material surface is being chipped away at the labs of Carnegie Melon University utilizing touchscreens that vibrate to travel shifting vibration frequencies that speak to our feeling of touch surfaces. (Bau et al., 2010). This cutting edge haptic gadget will be portrayed in detail later in the paper. (Israr, Bau, Kim, and Poupyrev, 2012).

\section{CUTANEOUS HAPTIC TECHNOLOGIES}

Numerous people who are visually impaired regularly get to data in content through screen perusers. With the appearance of cutting edge optical character acknowledgment (OCR) programming pages can be filtered and deciphered very quickly. Others may utilize electronic arrangements, for example, screen perusers related to voice synthesizers. One progressing issue for STEM understudies is getting to e.g., charts, because of a powerlessness to interpretation into the sound medium. Speaking to such graphically rich embellished detail on paper can be pricey, cumbersome, and hard to ship. One response to this issue is a material presentation. A material showcase is equipped for deciphering through its interface data on shape, surface, temperature, and hardness. The objective of utilizing a material presentation is create virtual items for the client to investigate with their feeling of touch. Be that as it may, innovations to achieve this undertaking are still in their early stages. The greatest boundaries are in making a gadget at the correct value point covering an enormous region with conservative surface forming actuators. As one can envision to get a decent goals in a material surface the thickness or populace per region of actuators must be reasonably packed. The following is a representation is given of how testing it is size savvy to fit this riddle together. A Braille zone of specks can get very jumbled and little actuators are required.

\section{MECHANICAL ACTUATED TECHNOLOGY}

BRAILLE SMARTPHONE: TED STAGE 2011 (Moore, 2013). : We have two groupings under this title. One is refreshable Braille Devices using pin actuated Braille cells of wither 6 or 8 pins. The other is purely tactile displays with an array of actuator driven pins to form a dimensional surface from a flat surface with a grid of pin holes. One novel attempt at bringing this technology into the homes of the blind community is a young engineer, Sumit Dugar, from India, who told his story of entrepreneurism on the TED stage in 2011. (Moore, 2013). He demonstrated the phone's capabilities noting that visually impaired users would be able to touch a real-time image with an advance tactile display of an individual on, e.g., a video chat and track with their tactile sense of touch changes in the person's expressions on their face. (Moore, 2013). The device is capable of helping a user interpret maps, play games, and other tactile abilities. (Moore, 2013) Below are two prototypes, the first being a tactile display for representing images and the second with a Braille refreshable display on its bottomside edge.

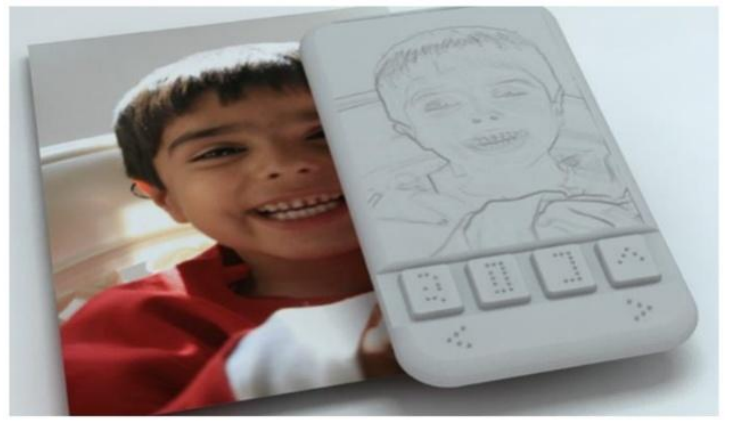

Figure 1: Using software an image is transformed into a tactile masterpiece of a transmitted image or video

(Moore, 2013)

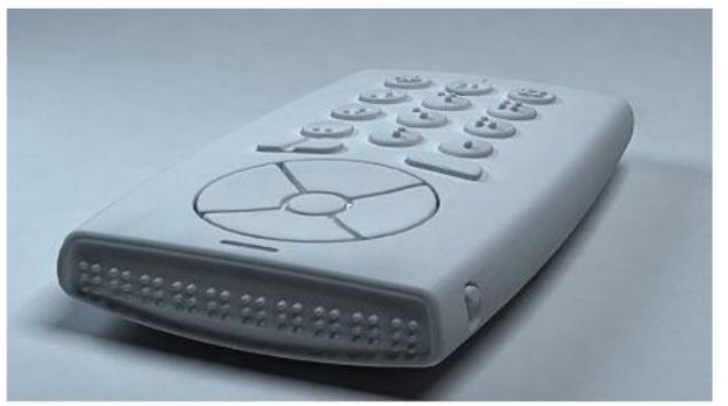

Figure 2: Smartphone with Braille display off bottom side again by entrepreneur extraordinaire Sumit Dugar (Larsen, 2013)

Figure2 demonstrates a cell phone for the outwardly debilitated with equipment that contains a complex lattice of little binds that go up and to suit content and pictures. (Larsen, 2013). It uses shape-memory amalgam innovation to grow or potentially agreement to its unique shape after use. The gadget exhibits up to 10 braille characters one after another. (Anderson, 2013). Frequently in the creating scene, cell phone clients use voice-based frameworks like Apple's VoiceOver (Larsen, 2013); be that as it may, because of the assortment of accents in the various tongues in India, these frameworks regularly don't work ideally. (Larsen, 2013). Then again, Braille remains the standard for openness for a significant part of the building up world's visually impaired populace, free of tongue transformation issues. A portion of its physical properties incorporate a numerical keypad has braille markings. An individual can enter braille letters.

\section{CONCLUSION}




\section{Haptic Structuring Assistive Innovation for Individuals Who Are Visually Impaired}

In this audit, a comprehension was created in learning the significance of individuals who are oblivious in regards to learn by material more than one sense to impart. For example, people who are visually impaired growing psychologically from just sound isn't suggested. Practicing our haptic faculties is basic in completely building up our subjective aptitudes as instructed by Barlow-Brown 2002. There is a wavering to suggest that an individual must gain just from Braille perusers as the innovation progresses (closely resembling iPad) since perusing from any material interface will do the trick. Additionally inspected was that it is so imperative to comprehend the structure of the hand/mind/body framework to ideally contribute new plans to the field of haptics. We found that one of the significant impediments in creating less expensive and increasingly dependable haptic presentations is through propelling improvements in actuator innovation, a segment at the core of the haptic showcase. One research at CMU keenly expelled the actuators issue from the image by building up another vibration based strategy to encode haptics information, e.g., surfaces, onto a level screen. Since the vibration innovation is still in its earliest stages it is smarter to not overlook the need to propel the actuator innovation. We found how significant in utilizing the common haptics framework in our bodies to give bits of knowledge into future advancements. Distinguishing work differences in the visually impaired network in the writing urges further motivating forces to grow new haptic plans to make people who are visually impaired progressively attractive for occupations. This audit examines the advantages and disadvantages of haptic innovation that incorporates: SmartTouch innovation, TeslaTouch Technology, Geomagic Phantom, .Geomagiccontact, Pure Form haptic presentation computer generated reality gadget, and HERMES/PC and Log-Text braille perusers. It was resolved by and large that these advances have incredible guarantee to conquer any hindrance between an out of reach web and an open one.. Looking back through separating down to the kinaesthetic and cutaneous haptic arrangements there are enormous issues to jump in kinaesthetic haptics is the engine control nuances that our bodies artfulness through. This I believe is because of an absence of more advanced sensor abilities. With cutaneous haptic gadgets the destruction is more in basics base material segment issues like the actuators. Actuator usefulness and size are the staying focuses in the structure and primarily because of makers experiencing difficulty machining little enough segments to work as one. We look at developing arrangement that influences financing to the different haptic activities. On the off chance that the administration demonstration soon, as it appears they may, motivating forces will be set up to beginning a course of movement that might be the push that this structure space required a few years back. This audit effectively overviewed an assortment of promising haptic structures that are spread over the plan range with blended impressions. Despite the fact that the ideas are there the equipment isn't. When the equipment gets up to speed with the product the field will be fit as a fiddle.

\section{REFRENCES}

1. Access Now, Inc. v. Southwest Airlines, Co, No. No. 02-21734-CIV 2271312 (Dist. Court, SD Florida 2002).

2. Liu, S. (2010). High electromechanical response electroactive polymers and their applications for solid state actuators. 3436168 Ph.D., The Pennsylvania State University, Ann Arbor.
3. Israr, A., Bau, O., Kim, S.-C., \& Poupyrev, I. (2012). Tactile feedback on flat surfaces for the visually impaired. Paper presented at the CHI '12 Extended Abstracts on Human Factors in Computing Systems, Austin, Texas, USA.

4. G. C. e. (2002). The Philippines disability survey: a collaborative survey. Department of Health and the University

5. of the Philippines.

6. America, B. A. o. N. (2012). The Evolution of Braille: Can the Past Help Plan the Future? A three-part article from the Braille Authority of North America.

7. Anderson, M. (2013). Inside the world's first braille cellphone [Resources_First Look]. Spectrum, IEEE, 50(7), 25-25.

8. Argyropoulos, V. S., \& Martos, A. C. (2006). Braille Literacy Skills: An Analysis of the Concept of Spelling. Journal of Visual Impairment \& Blindness, 100(11), 676-686.

9. Barlow-Brown, F., \& Connelly, V. (2002). The role of letter knowledge and phonological awareness in young braille readers. J Res Read, 25, 259-270.

10. Bau, O., Poupyrev, I., Israr, A., \& Harrison, C. (2010). TeslaTouch electrovibration for touch surfaces. Paper presented at the Proceedings of the 23nd annual ACM symposium on User interface software and technology, New York, New York, USA

11. Jan, J. E., B. Heaven, R. K., Matsuba, C., Beth Langley, M., Roman-Lantzy, C., \& Anthony, T. L. (2013). Windows into the Visual Brain: New Discoveries About the Visual System, Its Functions, and Implications for Practitioners. [Article]. Journal of Visual Impairment \& Blindness, 107(4), 251261.

12. Blanck, P. D., \& Sandler, L. A. (2000). ADA Title III and the Internet: Technology and civil rights. Mental \& Physical Disability L. Rep., 24, 855.

13. Braille.org. (2012). How many children in America are not taught to read?

14. Brewster, S., \& Brown, L. M. (2004). Tactons: structured tactile messages for non-visual information display. Paper presented at the Proceedings of the fifth conference on Australasian user interface Volume 28, Dunedin, New Zealand.

15. Brewster, S. B., Lorna M. (2004). Tactons: structured tactile messages for non-visual information display. Paper presented at the Proceedings of the fifth conference on Australasian user interface - Volume 28, Dunedin, New Zealand.

16. Burdea, G., \& Coiffet, P. (2003). Virtual reality technology. Presence: Teleoperators and virtual environments, 12(6), 663-664.

17. Burks, C. L. (2013). Improving Access to Commercial Websites under the Americans with Disabilities Act and the Twenty-First Century Communications and Video Accessibility Act Note. Iowa L. Rev., 99, 363-392.

18. Carnegie_Melon_Univeristy. (2014). Quality of Life \& Technology (QoLT). Web Page.

19. Chen, Y.-C., Chiang, C.-H., \& Chiu, H.-C. (2010). The recognition of 3D basic patterns and tactile icons for the blind. Paper presented at the Society for Social Management Systems (SSMS) International Symposium.

20. Hubel, D. H. (1995). Eye, brain, and vision. New York, NY, US Scientific American Library/Scientific American Books.

21. International, L. (2014). Visual Impairment Prevalence.

22. Jones, L. A., \& Lederman, S. J. (2006). Human hand function: Oxford University Press.

23. Kaczmarek, K. A. (2000). Electrotactile adaptation on the abdomen: preliminary results. Rehabilitation Engineering, IEEE Transactions on, 8(4), 499-505. doi: 10.1109/86.895953

24. Kaczmarek, K. A., Webster, J. G., Bach-y-Rita, P., \& Tompkins, W. J. (1991). Electrotactile and vibrotactile displays for sensory substitution systems. Biomedical Engineering, IEEE Transactions on, 38(1)

25. Kajimoto, H., Kawakami, N., Maeda, T., \& Tachi, S. (2004) Electro-tactile display with tactile primary color approach. Paper presented at the Proceedings of International Conference on Intelligent Robots and Systems.

26. Kuber, R., Yu, W., O, M. S., \& Modhrain. (2011). Evaluation of Haptic HTML Mappings Derived from a Novel Methodology. ACM Trans. Access. Comput., 3(4), 1-28. doi: 10.1145/1952388.1952389

27. Agelii, M., \& Rönnbäck, A. (1996). Teaching braille to beginners by using a computer. COLLOQUESINSTITUT NATIONAL DE LA SANTE ET DE LA RECHERCHE MEDICALE COLLOQUES ET SEMINAIRES, 37-44.

28. Bigelow, A. (1987). Early words of blind children. J Child Lang, 14, 47-56.

\section{Published By:}

Blue Eyes Intelligence Engineering and Sciences Publication

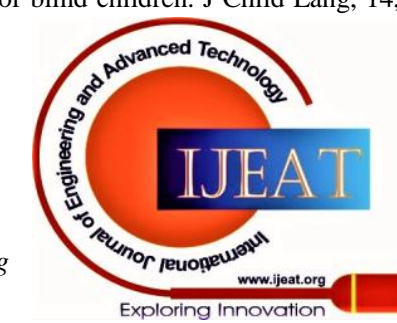


29. Kuber, R., Yu, W., \& O’Modhrain, M. S. (2011). Evaluation of haptic html mappings derived from a novel methodology. ACM Transactions on Accessible Computing (TACCESS), 3(4), 12.

30. Kuber, R., Zhu, S., Arber, Y., Norman, K., \& Magnusson, C. (2014a) Augmenting the non-visual web browsing process using the geomagic touch haptic device. SIGACCESS Access. Comput.(109), 410. doi: 10.1145/2637487.2637488

31. Kuber, R., Zhu, S., Arber, Y., Norman, K., \& Magnusson, C. (2014b). Augmenting the non-visual web browsing process using the geomagic touch haptic device. ACM SIGACCESS Accessibility and Computing(109), 4-10.

32. Landwehr, A. (2010). Amending the digital divide. Syracuse Sci. \& Tech. L. Rep., 2010, 90-162.

33. Larsen. (2013). World's first Braille smartphone in development. CNET Tech Culture.

34. Leung, R., MacLean, K., Bertelsen, M. B., \& Saubhasik, M. (2007). Evaluation of haptically augmented touchscreen gui elements under cognitive load. Paper presented at the Proceedings of the 9th international conference on Multimodal interfaces, Nagoya, Aichi, Japan.

35. Lévesque, V. Blindness, technology and haptics.

36. ADA. (1990). Americas Disability Act PART 36-NONDISCRIMINATION ON THE BASIS OF DISABILITY BY PUBLIC ACCOMMODATIONS AND IN COMMERCIAL FACILITIES t.

37. Xiaosong, W., Seong-Hyok, K., Haihong, Z., Chang-Hyeon, J., \& Allen, M. G. (2012). A Refreshable Braille Cell Based on Pneumatic Microbubble Actuators. Microelectromechanical Systems, Journal of, 21(4), 908-916. doi: 10.1109/JMEMS.2012.2190043

38. Xie, X., Zaitsev, Y., Vel?squez-Garc?a, L. F., Teller, S. J., \& Livermore, C. (2014). Scalable, MEMS-enabled, vibrational tactile actuators for high resolution tactile displays. Journal of Micromechanics and Microengineering, 24(12), 125014.

39. Xu, C., Israr, A., Poupyrev, I., Bau, O., \& Harrison, C. (2011). Tactile display for the visually impaired using TeslaTouch. Paper presented at the CHI '11 Extended Abstracts on Human Factors in Computing Systems, Vancouver, BC, Canada.

40. Yu, W., Guffie, K., \& Brewster, S. (2001). Image to haptic data conversion: A first step to improving blind people's accessibility to printed graphs. Paper presented at the Proceedings of Eurohaptics.

41. Yu, W., Ramloll, R., \& Brewster, S. (2001). Haptic graphs for blind computer users. In S. Brewster \& R. Murray-Smith (Eds.), Haptic Human-Computer Interaction (Vol. 2058, pp. 41-51): Springer Berlin Heidelberg.

\section{AUTHORS PROFILE}

Vivek Agrawal, Student, B.Tech. Vellore Institute of Technology, Vellore agrawal.vivek2017@gmail.com

Vishakha Singh, Student, B.Tech. Vellore Institute of Technology, Vellore vishakha.singh1905@gmail.com

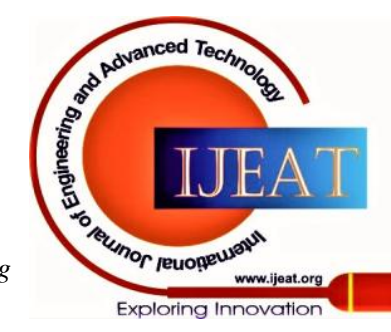

